\title{
Protein Attributes Microtuning System (PAMS): an effective tool to increase protein structure prediction by data purification
}

\author{
Fan Zhang ${ }^{\S}$, David Povey ${ }^{* * *}$, Paul Krause ${ }^{f f}$ \\ University of Surrey, Guildford, GU2 7XH, UK.
}

\begin{abstract}
Given the expense of more direct determinations, using machine-learning schemes to predict a protein secondary structure from the sequence alone remains an important methodology. To achieve significant improvements in prediction accuracy, the authors have developed an automated tool to prepare very large biological datasets, to be used by the learning network. By focusing on improvements in data quality and validation, our experiments yielded a highest prediction accuracy of protein secondary structure of $90.97 \%$. An important additional aspect of this achievement is that the predictions are based on a template-free statistical modeling mechanism. The performance of each different classifier is also evaluated and discussed.

In this paper a protein set of 232 protein chains are proposed to be used in the prediction. Our goal is to make the tools discussed available as services in part of a digital ecosystem that supports knowledge sharing amongst the protein structure prediction community.
\end{abstract}

Index Terms-Automata, Biomedical Computing, Proteins, Prediction Methods, Data Management.

\section{BACKGROUND INTRODUCTION}

$\mathrm{P}$ ROTEINS carry out vital biological functionalities for each living creature. To correctly enable this functionality, a protein must fold into a unique three-dimensional shape in a moist environment. Errors in protein folding usually cause failures in performance of biological functions, and these errors are implicated in the early stages of deadly diseases such as amyloidoses [1], Alzheimer's disease [2], prion diseases [3], and most cancers [4]. Clearly, further understanding of protein structural characteristics, will help the pharmaceutical industry to may develop medicines to cure or even prevent these diseases.

There are various ways to determine protein structure. X-Ray crystallography [5] and Nuclear Magnetic Resonance (NMR) spectroscopy [6] are the most frequently used techniques to empirically determine the atomic model of the protein, and are widely used in academic institutes and the pharmaceutical industry. However, these techniques are both time-consuming,

\footnotetext{
${ }^{\S}$ Email:f.zhang@surrey.ac.uk, collaborated project between Department of Computing and Division of Chemistry in University of Surrey.

Email:d.povey@surrey.ac.uk, Professor in Computer Aid Chemistry, Division of Chemistry, University of Surrey.

t† Email:p.krause@s surrey.ac.uk, Professor in Software Engineering, Department of Computing, University of Surrey.
}

and expensive. Furthermore, specialised knowledge is required to apply these techniques, making them even more difficult to be used by other scientists. An alternative methodology is to predict protein structure based on the application of machine learning schemes to existing biological data. These techniques are gaining significant attention because of their potential low cost and ease of application.

In the early 1960s Christian Anfinsen and his colleagues discovered that the ribonuclease protein denatured while the chemical environment was changed by either heat, or adding certain chemicals. The denatured ribonuclease folded back to its natural three dimensional shapes after the temperature was lowered or the certain chemicals were removed [7]. His concern of connection between the amino acid sequence and the biological active conformation laid out the foundation of in silico prediction of protein structure from the study of characteristics of sequence.

Machine learning techniques for studying and predicting protein structure have been under development for the past three decades. However these procedures suffered from the lack of the computational capacity and the limited availability of protein structure data. Along with the development of computer hardware and the growth of online protein data resources, the future of in silico prediction of the protein structure seems to be more and more promising.

There are several categories of prediction methodologies, which vary from each other by processed data. The homology modelling methods produce accurate predictions on proteins that share more than $70 \%$ sequence identity, but for sequences that share less than $25-30 \%$ sequence similarity, the homology models may fail [8]. The latter are frequently used to provide starting structures for molecular replacement in X-Ray crystallography [9]. Protein threading techniques [10] search through currently known structures and identify the one which is most likely to be appropriate for the protein sequence undr investigation. Unlike the homology modelling tools the threading methods deal with the proteins that share no obvious sequence identities, but have approximately similar folds. A recent example of protein threading application is called "Wurst" [11]. Statistical template-free methods predict the secondary structure subtype from the amino acid residue characteristics alone. There is no obvious template available in these sequences. In our research, the predictions are based on a template-free statistical modelling mechanism. 
The problem of protein structure prediction has been tackled for the past four decades, starting with methods such as the single residue statistical approach [25], often categorized as the first generation methodology. The first generation method produced approximately 50\% prediction result and later on, along with the advancement of machine learning techniques and the further understanding of feature descriptors describing the characteristics of amino acid residues, the predictive accuracy reached nearly $80 \%$. By using the PHD [27] method an average of $76 \%$ of amino acid residues are correctly predicted, while the JPRED [28, 29] yielded the best prediction accuracy of $76.4 \%$. The PSIPRED [30] achieved average accuracy between $76.5 \%$ and $78.3 \%$. The BAYESPROT [26] reported a highest prediction accuracy of $76.8 \%$.

The mission in this paper is to explore an alternative way to improve protein secondary structure prediction accuracy by purifying the applied biological data resources, instead of producing a novel machine-learning scheme. In this paper the authors present an effective tool - PAMS - to process the various available biological data resources, and then proceed to the prediction of the secondary structure. The tool has the capability to run through vast amount of experiments based on simple instructions. Currently five million learning and prediction tasks have been carried out in a distributed computational platform. The human intervention in this process is significantly low.

In this research, PAMS produced a highest secondary structure prediction accuracy of $90.97 \%$. Apart from this significant achievement, we report a number of additional results of interest. Firstly, an FD232 ${ }^{\ddagger \ddagger}$ dataset has been collated, which we have found to be a good candidate protein sample set for protein secondary structure prediction. Next, a wide range of amino acid feature descriptors are assessed and ranked according to their performances. Furthermore, we found that after the window size reaches 21 , which is believed to be an optimal window size, the protein structure prediction accuracies do not vary radically if the window size is increased further. Finally, a list of machine learning classifiers is assessed according to their prediction accuracy and efficiency. Here we found that instance based classifiers [31] were capable of producing good prediction accuracies, but were not efficient. In contrast, the Tree-Augmented naïve Bayesian network classifier classifiers/Forest-Augmented naïve Bayesian network classifier (FAN/TAN) [21], were capable of predicting protein secondary structure with an accuracy of $81 \%-84 \%$, but finish the task in a reasonably short time ( 3 seconds -1 minute, depend on the size of the dataset). Apart from these solid results, we also raise a number of research questions at the end of the paper.

The tools discussed in this paper are being progressively made available as web-services. A sub-goal of our research is to support the creation of a digital-ecosystem for the Protein Structure Prediction community. Once our tools are available

\footnotetext{
$\ddagger \ddagger$ A dataset generated in this research, contains 232 protein chains. Send an email to the author to ask for a copy.
}

as services, they may be composed with other services in the network to provide a configurable and extensible framework for the research community.

\section{System ANalysis And Design of The PAMS}

PAMS is a standalone tool for preparing training and testing sets for machine learning classifiers. It is coded in Java, deployed and tested on a Red Hat Linux system. The Red Hat operation system is a reliable and well supported developing platform, which is widely used in the bioinformatics research arena. PAMS accesses a MySQL database [12], to retrieve data resources and deposit experiment results.

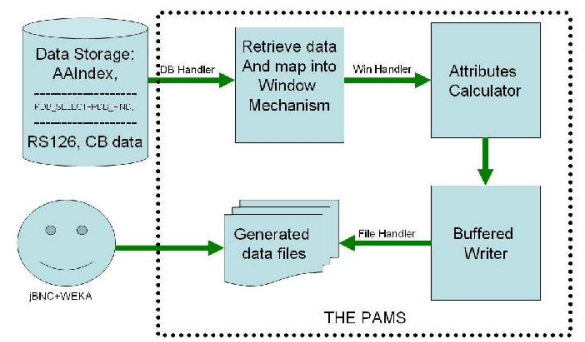

\section{II-1: The PAMS infrastructure}

As described in II-1, the software contains four major modules in the infrastructure. The DB Handler retrieves data from the database, and then transmits them to be mapped in a window.

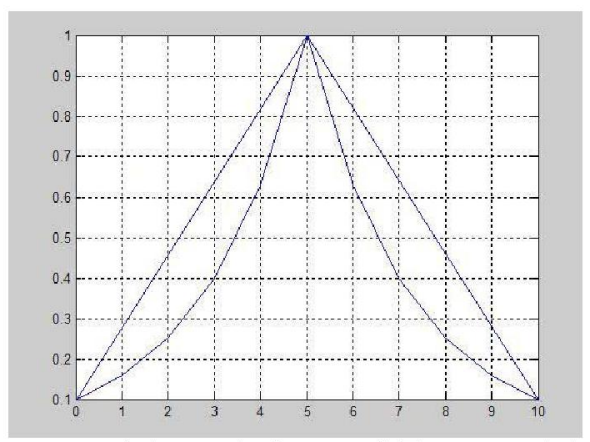

II-2: Linear weight variation and Exponential weight
variation

There are two weight variation models implemented in this paper, as described in figure II-2. The straight line represents the linear weight variation model, and the curve represents the exponential weight variation model. It is assumed that the window buffer contains $\mathrm{N}$ amino acids, the set $\left\{W_{0}, W_{1}, \ldots W_{N-1}\right\}$ indicates weight values assigned to each amino acid according to its position. The function to calculate the linear weight value for the amino acid in position $i$ is:

$$
W_{i}=W_{(N-1) / 2}-\frac{2 \times|(N-1) / 2-i| \times\left(W_{(N-1) / 2}-W_{0}\right)}{N-1}
$$

And the function to calculate the exponential weight value for the amino acid in position $i$ is: 


$$
W_{i}=e^{\frac{\left(\left|-\frac{N-1}{2}\right|\right) * \log ^{W_{0}}}{\frac{N-1}{2}}}
$$

The choice of linear or exponential weight variation models is currently a matter of judgment. The weight assigned to an amino acid represents the degree of influences of that particular amino acid on the central amino acid. Further work is needed on defining objective criteria for identifying the optimal function to be used to represent the degree of influences to the central amino acid, but this is outside the scope of this paper.

The database of the PAMS infrastructure contains several available biological data resources. The Protein Data Bank (PDB) [13] contains 39853 records of protein structure, with 33718 of them structured by X-Ray crystallography, 5914 of the samples' structure determined by the NMR spectroscopy. Each record entry contains a list of three-dimensional coordinates of each atom of that particular protein, therefore presents the shape of the protein.

The PDB SELECT25 $[14,15]$ contains 3080 protein chains, with 459963 amino acid residues, which are selected from the current Protein Data Bank to be the representative data set. Each pair of chains of this dataset share less than $25 \%$ sequence identity, hence the data set is considered as a collection of protein entries without strong homological correlations among them.

The DSSP [16] database is a database of secondary structure assignments for protein entries of the PDB. The database contains the sequence, the corresponding secondary structure of proteins, and further information about the particular protein. The sequences and secondary structures of the protein samples used in this paper were extracted from DSSP according to the protein identities, which are defined in PDB_SELECT25. The extracted information contains eight secondary structure subtypes, e.g. the set of $\{H, E, B, G, I, T, S,-\}$. To simplify the prediction problem, a " $\mathrm{H}, \mathrm{G}$, and $\mathrm{I}$ to $\mathrm{H}$; $\mathrm{E}$ to $\mathrm{E}$, the rest to $\mathrm{C}$ " eight-to-three state reduction method is applied to assign each amino acid residue's secondary structure type to be one of the set of $\{C, H, E\}$.

The AAIndex $[17,18,19]$ database published by the Japanese Genome project contains 516 characteristic descriptors of each amino acid residue, which are collected from the literature. There are physical, chemical attributes of each amino acid within the database, and statistical analyses of each amino acid residues' structural propensities. The entries from the AAIndex database were used to represent the features of each amino acid residue. Ten sets of residue characteristics were removed due to their containing undetermined values.

This use of available resources is an advantage of this research, saving much time and labour.

The Weka [20] machine learning toolbox is used to perform the learning and prediction tasks for the study. Weka supports a set of machine learning classifiers, including decision tree based classifiers [22,32], instance based classifiers [31], neural network classifiers, and Bayesian network classifiers [21]. Weka project is especially good for bioinformatics study [23].
The training and testing data sets have to be prepared in the ARFF [20] file format, in order to be processed by the Weka classifiers toolbox. Another advantage for us is that Weka is coded in Java, and so easily integrated into the PAMS software framework. In addition, Weka contains tools for data pre-processing, regression, clustering, and visualization [20].

The measurement of the Weka is the cross-validation measurement, which is a standard method to estimate classification accuracy over unseen data. For N-fold cross validation, the data are split into $\mathrm{N}$ subsets. One subset is then used as a testing set and the rest are combined together as the training set. This classification is performed $\mathrm{N}$ times, and the accuracies averaged. By default, all measurements used in this research are 10 -fold cross validations.

The datasets processed in this research are discretized before being sent into the classifiers. The process of discretization converts the numerical attributes into nominal (categorical) attributes, and decreases the computational complexity [24].

\section{EXPERIMENTS PERFORMED BY THE PAMS}

A series of experiments are performed to explore the correlations between the amino acid residues of a particular protein and their corresponding secondary structure types. A number of additional experiments are performed to gain further understanding of the factors that influence protein structure prediction accuracies.

\section{A. Selection of Protein Samples}

To perform a prediction, a list of known protein entries has to be provided. The prediction of the protein structure is then based on the knowledge that the learning network discovered from the protein samples with known structure. Apart from the homological modelling, where selected proteins share homological characteristics, in this study the 3080 protein samples share no obvious homological commons. However the use of 3080 protein entries is not effective due to the learning process needing to go through a large amount of residues. To select a smaller and more representative protein sample set, each protein entry of the 3080 is tested with all the 506 attributes, which are combined together, and the performance of that particular protein is recorded. In the following plot III-1, the $\mathrm{X}$ axis indicates the length of the particular protein sample, and the $\mathrm{Y}$ axis represents the self-test prediction accuracy of the designated protein sample: 


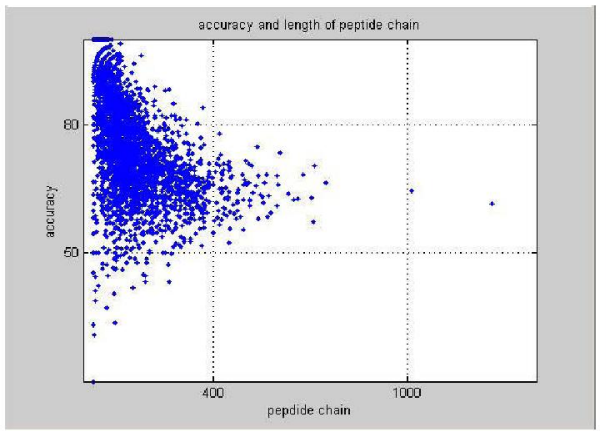

III-1: 3080 protein entries plotted as accuracy and peptide chain length.

The selection of the protein entries provides an alternative way to determine the quality of these proteins, which are participants in the process of protein secondary structure prediction. 232 peptide chains (including 11760 amino acid residues) are selected from the above diagram. The selection is based on considerations of both high self-test accuracy, and representativeness to various lengths of the peptide chain.

\section{B. Selection of Window Mechanism}

Windowing mechanisms have been applied since 1980s [33, $34,35]$. The window moderates neighbouring amino acids' influences on the central amino acid's secondary structure. The critical problem is to determine an optimal window mechanism by selecting the length of the window and weight variation models. There simplest weight variation models are the linear and exponential weight variation models, as presented in the diagram II-l. We started our study with these.

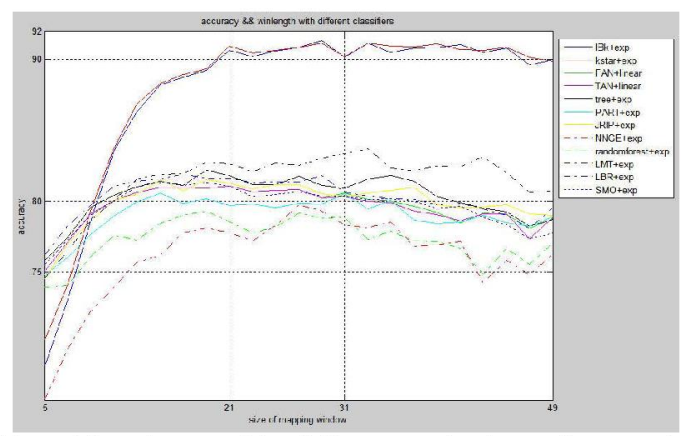

III-2: Classifications with different window size and weight variation model.

In diagram III-2, each curve indicates a situation of using one particular classifier with either a linear or an exponential weight variation model. From the plot, it is quite obvious that as the window length increase from 5 to 21 , the prediction accuracies rise. After that, the prediction accuracies tend to stay stable. For some classifiers, the prediction accuracies dropped a little bit, and for most of the classifiers, the variation of the curve after the window length reach 21 could be ignored. From the diagram, nearly all the classifiers report highest accuracies when the window length is set to 21 .

Both the exponential and linear weight variation models are tested in this experiment. The overall performance of various learning schemes with various window length and weight variation models is shown in Table III-1:

\begin{tabular}{l|l|l|l|l|l|l|c} 
& $\begin{array}{l}\text { Naive } \\
\text { Bayes }\end{array}$ & FAN/TAN & AODE & $\begin{array}{l}\text { RBF } \\
\text { Network }\end{array}$ & B1 & Bk & KStar \\
\hline Linear & $74.62 \%$ & $81.14 \%$ & $81.88 \%$ & $80.70 \%$ & $90.50 \%$ & $90.67 \%$ & $90.97 \%$ \\
\hline Exp & $75.45 \%$ & $81.74 \%$ & $82.36 \%$ & $80.88 \%$ & $87.67 \%$ & $88.30 \%$ & $88.51 \%$ \\
\hline & ID3 & SMO & $\begin{array}{l}\text { Random } \\
\text { Forest }\end{array}$ & $\begin{array}{l}\text { Decision } \\
\text { Table }\end{array}$ & NNGE & PART & LBR \\
\hline Linear & $80.20 \%$ & $82.67 \%$ & $85.60 \%$ & $81.40 \%$ & $83.94 \%$ & $82.10 \%$ & $82.65 \%$ \\
\hline Exp & $82.83 \%$ & $82.36 \%$ & $84.32 \%$ & $81.73 \%$ & $81.89 \%$ & $80.46 \%$ & $81.95 \%$
\end{tabular}

Table III-1: The performance table of machine learning schemes with linear and exponential weight variation models.

The instances based classifiers report the highest prediction accuracy when a linear weight variation model is applied. However, the classification may take days to finish. Using the FAN/TAN classifiers is the most efficient, taking less than three seconds in a standard Linux operation system. The prediction accuracy is approximately $81-82 \%$.

These results imply that instances based classifiers should be used when the prediction accuracy is paramount. FAN/TAN can be used if preliminary results are required in a short time, e.g. in a situation where a vast amount of experiments wait to be carried out.

\section{Ranking the Amino Acid Feature Descriptors}

There are 506 feature descriptors that could be used in the process of protein secondary structure prediction. Obviously using all the feature descriptors in the prediction is not economic, leading to time-consuming tasks. We thus need to optimise the selection of feature descriptors.

There are various ways to rank the feature descriptors. In this study each individual feature descriptor is applied to all of the 3080 protein samples, and ranked by the self-test results. The top ten ranked feature descriptors are listed in the following table, by applying the instance based classifiers:

\begin{tabular}{c|c} 
Feature Descriptor ID & Self-Testing Accuracy \\
\hline PTIO830101 & $55.40 \%$ \\
\hline MUNV940102 & $55.32 \%$ \\
\hline ROBB760103 & $55.22 \%$ \\
\hline AURR980113 & $55.16 \%$ \\
\hline SUEM840101 & $55.00 \%$ \\
\hline KANM800103 & $54.93 \%$ \\
\hline AURR980109 & $54.90 \%$ \\
\hline QIAN880107 & $54.86 \%$ \\
\hline MUNV940101 & $54.83 \%$ \\
\hline RACS820108 & $54.81 \%$ \\
Table III-2: Top ten ranked feature descriptors.
\end{tabular}

Although the process of prediction is time consuming, these 10 feature descriptors are still tested by KStar, IBk, Decision Tree, Bayesian network classifiers. The produced ranks are similar to the table listed above.

The top 168 ranked feature descriptors by applying the TAN classifier is clustered and illustrated in the following table:

\begin{tabular}{c|c} 
Accuracy range & Numbers of Attributes \\
\hline$>=55.0 \%$ & 5 \\
\hline$>=54.0 \% \& \&<=55.0 \%$ & 28 \\
\hline$>=53.0 \% \& \&<=54.0 \%$ & 36
\end{tabular}




\section{\begin{tabular}{c|c}
$>=52.0 \% \& \&<=53.0 \%$ & 31 \\
\hline$>=51.0 \% \& \&<=52.0 \%$ & 32
\end{tabular} \\ Table III-3: Top 168 ranked feature descriptors}

\section{Selection of the Number of Applied Feature Descriptors:}

This experiment explores how the numbers of applied feature descriptors influence the prediction accuracy. We are not aware of any existing results on the correlation between the numbers of feature descriptors applied and the prediction results. We can gain clear computational benefits if decreasing the number of feature descriptors does not pull down the prediction accuracy drastically.

In the following diagram, the experiments are tested on the 232 dataset, with the TAN classifier applied. Through the diagram, the prediction accuracies do not vary radically while the numbers of applied feature descriptors are changed. The questions now is, which specific feature combinations should be used?

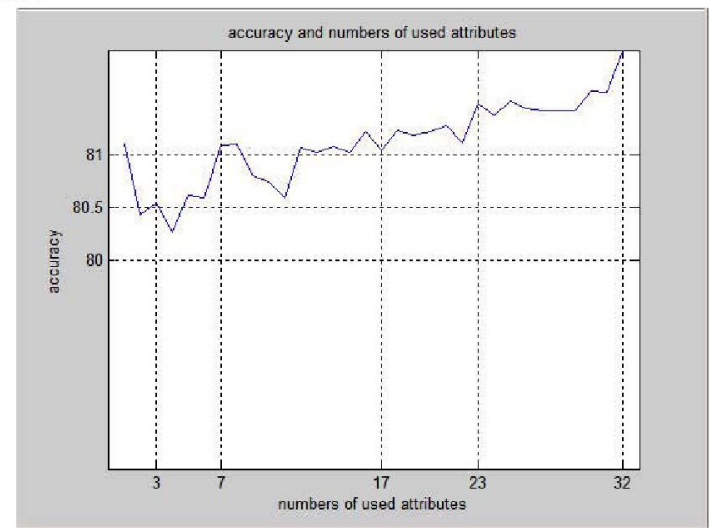

III-3: accuracy and numbers of applied feature descriptors.

\section{E. Selection of Combinations of Feature Descriptors:}

Selection of an optimal combination of feature descriptors is vital for accurate prediction of protein secondary structure. A total of 506 feature descriptors could be used, giving a vast number of potential combinations. We used a cluster of over 100 processors to provide sufficient computational capacity for these experiments.

Firstly, combinations of two random feature descriptors from the 506 sets are tested. Then random selections of combinations of any three feature descriptors from the top 168 feature descriptors are tested. In general over five million tasks have been carried out.

Among these, a total of 139 combinations of feature descriptors are categorized to be good quality combinations with corresponding prediction accuracies of greater than $84 \%$. The diagram III-4 shows the appearance of several particular feature descriptors among these combinations. Several interesting results are pertinent. Notice that feature descriptor "BLAM930101" appears in almost all of the good quality predictions. This potentially means that the physical/chemical characteristics that the feature descriptor "BLAM930101" represents, may have a direct impact on the protein secondary structure, and could easily be spotted by the classifiers used in this research. Another interesting result is that the feature descriptors "BLAM930101" and "QIAN880132" both feature in one third of the results. This phenomenon may mean that the characteristics of these two feature descriptors also have greater influences on the secondary structure of the amino acid than other feature descriptors - suggesting they may dominate any predictions in which they are involved.

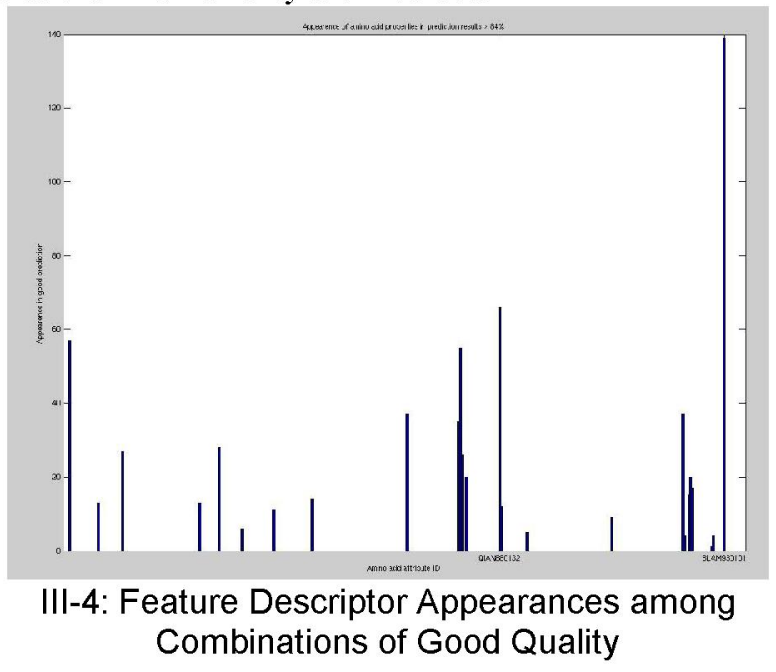

Similarly, the appearances of the feature descriptors among the combinations with poor predictive quality are presented in the following plot:

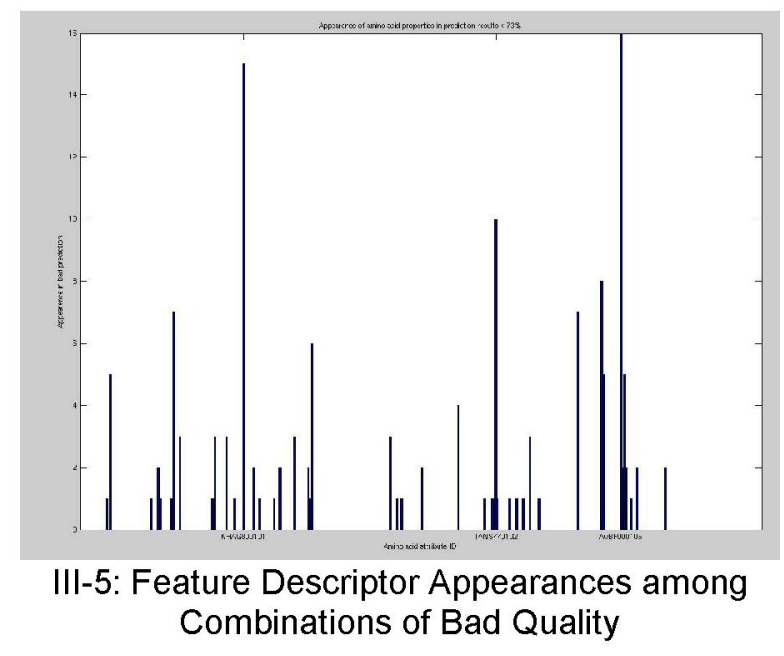

\section{CONClusion}

In general, the PAMS tool is an effective tool for providing easy access to generate the desired datasets as and when a user wants. The above experiments significantly extend understanding of the machine learning schemes that participate in protein structure prediction activities. The instance-based classifiers provide highest accuracy, but TAN/FAN classifiers are significantly more efficient and hence suitable for use in experiments containing vast amount of tasks. 232 protein samples are selected to be both representative to the PDB databank and "good quality" in predicting protein secondary structure in a template-free modelling manor. The window size 
of 21 is thought to be the optimal window size. The superiorities of the weight variation models are connected to both the implemented classifiers and applied data sets. Finally, the PAMS provides the basis for a unified tool for learning and prediction.

\section{FUTURE WORK OF THE PAMS}

In the further study, a deeper study about the weight variation model for the window mechanism is planned, to gain further understanding of how the adjacent amino acid residues affect the central amino acid's secondary structure type. More combinations of the amino acid feature descriptors will also be examined. Two additional development tasks are in hand for PAMS. Firstly, an automatic update module is required to retrieve online biological data resources. Secondly, the PAMS module could be migrated to web interface, enabling people around the world to configure it and generate the desired dataset, thus providing a foundation for the digital-ecosystem discussed in the introduction.

\section{ACKNOWLEDGMENT}

We would like to thank Dr Lee Gillam and Mr. Gary Dear in Department of Computing in University of Surrey, support with the computer grid; Dr Brendan Howlin and Dr Ian Hamerton in Division of Chemistry of University of Surrey, for helpful discussion, Mr. Chris Bradshaw in Division of Chemistry for IT support.

\section{REFERENCES}

[1] E. M. Brunt and D. G. Tiniakos, "Metabolic storage diseases: amyloidosis," Clin. Liver Dis., vol. 8, no. 4, pp. 915-30, x, Nov. 2004.

[2] R. Taylor, "Evolutions: Brain imaging," The Journal of NIH Research, May 1990, Vol. 2, p. 103.

[3] R. T. Johnson, "Prion diseases," Lancet Neurol., vol. 4, no. 10, pp. 635-642, Oct. 2005 .

[4] R.W. Moss, Galen on Cancer - How Ancient Physicians Viewed Malignant Disease, 1989.

[5] J. Drenth, Principles of Protein X-Ray Crystallography. Springer-Verlag Inc. NY: 1999, ISBN 0-387-98587-5.

[6] J.W. Akitt; B.E. Mann, NMR and Chemistry. Stanley Thornes. Cheltenham, UK, 2000.

[7] C. B. Anfinsen, "Principles that govern the folding of protein chains," Science, vol. 181, no. 96, pp. 223-230, July1973.

[8] B. Rost, "Review: protein secondary structure prediction continues to rise," J. Struct. Biol., vol. 134, no. 2-3, pp. 204-218, May2001.

[9] B. Rost and S. O'Donoghue, "Sisyphus and prediction of protein structure," Comput. Appl. Biosci., vol. 13, no. 4, pp. 345-356, Aug.1997.

[10] Bowie, J. U.; Luthy, R.; and Eisenberg, D. 1991. "A method to identify protein sequences that fold into a known three-dimensional structure." Science 253:164-170.

[11] Torda AE, Procter JB \& Huber T (2004) "Wurst: a protein threading server with a structural scoring function, sequence profiles and optimized substitution matrices." Nucleic Acids Res 32, W532--W535.

[12] MySQL Co. Ltd. http://www.mysql.com [Access Date: 07/10/2006]

[13] H.M. Berman, J. Westbrook, Z. Feng, G. Gilliland, T.N. Bhat, H. Weissig, I.N. Shindyalov, P.E. Bourne: The Protein Data Bank. Nucleic Acids Research, 28 pp. 235-242 (2000).

[14] U.Hobohm, M.Scharf, R.Schneider, C.Sander: "Selection of a representative set of structures from the Brookhaven Protein Data Bank", Protein Science 1 (1992),409-417.

[15] U.Hobohm, C.Sander: "Enlarged representative set of protein structures", Protein Science 3 (1994) 522.
[16] W. Kabsch and C. Sander, "Dictionary of protein secondary structure: pattern recognition of hydrogen-bonded and geometrical features," Biopolymers, vol. 22, no. 12, pp. 2577-2637, Dec.1983.

[17] S. Kawashima, H. Ogata, and M. Kanehisa, "AAindex: Amino Acid Index Database," Nucleic Acids Res., vol. 27, no. 1, pp. 368-369, Jan. 1999.

[18] K. Tomii and M. Kanehisa, "Analysis of amino acid indices and mutation matrices for sequence comparison and structure prediction of proteins," Protein Eng, vol. 9, no. 1, pp. 27-36, Jan.1996.

[19] K. Nakai, A. Kidera, and M. Kanehisa, "Cluster analysis of amino acid indices for prediction of protein structure and function," Protein Eng, vol. 2, no. 2, pp. 93-100, July1988.

[20] Ian H. Witten and Eibe Frank (2005) "Data Mining: Practical machine learning tools and techniques", 2nd Edition, Morgan Kaufmann, San Francisco, 2005.

[21] R. Bouckaert, "Bayesian Network Classifiers in Weka", Technical Report, Department of Computer Science, Waikato University, Hamilton, NZ 2005.

[22] C.S. Lin, T. Smith, "A tree-based algorithm for predicate-argument recognition." Association for Computing Machinery New Zealand Bulletin, 2(1): online journal, January 2006.

[23] E. Frank, M. Hall, L. Trigg, G. Holmes, and I. H. Witten, "Data mining in bioinformatics using Weka," Bioinformatics., vol. 20, no. 15, pp. 2479-2481, Oct.2004.

[24] U.M. Fayyad, and K.B. Irani. Multi-Interval Discretization of Continuous-Valued Attributes for Classification Learning. Proc. of 13th Int. Joint Conf. on AI, 1993.

[25] P. Y. Chou and G. D. Fasman, "Prediction of protein conformation," Biochemistry, vol. 13, no. 2, pp. 222-245, Jan. 1974.

[26] A. Chinnasamy, W. K. Sung, and A. Mittal, "Protein structure and fold prediction using Tree-Augmented naive Bayesian classifier," J. Bioinform. Comput. Biol, vol. 3, no. 4, pp. 803-819, Aug.2005.

[27] B. Rost and J. Liu, "The PredictProtein server," Nucleic Acids Res., vol. 31, no. 13, pp. 3300-3304, July2003.

[28] J. A. Cuff and G. J. Barton, "Application of multiple sequence alignment profiles to improve protein secondary structure prediction," Proteins, vol. 40, no. 3, pp. 502-511, Aug.2000.

[29] J. A. Cuff, M. E. Clamp, A. S. Siddiqui, M. Finlay, and G. J. Barton, "JPred: a consensus secondary structure prediction server," Bioinformatics., vol. 14, no. 10, pp. 892-893, 1998.

[30] D. T. Jones and J. J. Ward, "Prediction of disordered regions in proteins from position specific score matrices," Proteins, vol. 53 Supp1 6, pp. 573-578, 2003.

[31] Cleary J.G. and Trigg L.E. (1995) "K*: An Instance-Based Learner Using an Entropic Distance Measure," Proc Machine Learning Conference, Tahoe City, CA, USA, pp. 108-114.

[32] J.R. Quinlan, "C4.5: Programs for machine learning," San Mateo, CA: Morgann Kaufmann.

[33] D. Przybylski and B. Rost, "Alignments grow, secondary structure prediction improves," Proteins, vol. 46, no. 2, pp. 197-205, Feb.2002.

[34] N. Qian and T. J. Sejnowski, "Predicting the secondary structure of globular proteins using neural network models," J. Mol. Biol., vol. 202, no. 4, pp. 865-884, Aug. 1988.

[35] B. Rost, C. Sander, and R. Schneider, "Redefining the goals of protein secondary structure prediction," J. Mol. Biol., vol. 235, no. 1, pp. 13-26, Jan. 1994. 